\title{
OSCILLATIONS IN MAGNETORESISTANCE AND INTERLAYER COUPLING IN MAGNETIC SANDWICH STRUCTURES
}

\author{
J. BARNAŚ \\ Institute of Physics, A.M. University, Matejki 48/49, 60-769 Poznan, Poland \\ and \\ Laboratory of Solid State Physics and Magnetism, Catholic University of Leuven \\ Celestijnenlaan $200 \mathrm{D}, 3001$ Leuven, Belgium \\ AND B. BULKA \\ Institute of Molecular Physics, Polish Academy of Sciences, \\ Smoluchowskiego 17, 60-179 Poznań, Poland
}

Kubo formalism is used to calculate the magnetoresistance due to magnetization rotation in a structure consisting of two magnetic films separated by a nonmagnetic layer. In the approximation of a uniform relaxation time for each layer, the oscillatory term in magnetoresistance corresponds to the oscillation period which depends on the potential barriers at the interfaces. This period is longer than the oscillation period observed in the coupling parameter.

PACS numbers: 72.15.Gd, 73.40.Jn

The oscillatory interlayer coupling in magnetic multilayers [1] is of quantum origin and is usually described either in terms of the Ruderman-Kittel-KasuyaYoshida theory [2], or within the spin-dependent quantum-well models [3]. In both approaches the oscillations result from spin dependent interference of electron waves and the oscillation periods are determined by the extremal spanning vectors of the appropriate Fermi surface.

On the contrary, the giant magnetoresistance (GMR) effect [4] is not of quantum origin and therefore there is no direct link between the interlayer coupling phenomenon and GMR, except that in many cases the antiferromagnetic coupling is used to obtain the antiparallel alignment of the film magnetizations. However, the interference effects responsible for the oscillatory coupling can also give rise to an oscillatory contribution to the GMR effect $[5,6]$. In the free-electron-like model and without taking into account the aliasing effect, there is a single oscillation period $\Lambda_{\mathrm{c}}$ in the coupling parameter, $\Lambda_{\mathrm{c}}=\lambda_{\mathrm{F}} / 2$, where $\lambda_{\mathrm{F}}$ is the Fermi wavelength. The same oscillation period also occurs in GMR. Consequently, the oscillations 
in GMR and interlayer coupling corresponding to the oscillation period $\Lambda_{\mathrm{c}}$ are then correlated [7]. However, there are additional oscillations in GMR, with the oscillation period longer than $\Lambda_{\mathrm{c}}$ and dependent on the potential barriers at the interfaces. In this paper we concentrate just on those long-period oscillations in GMR.

We consider two ferromagnetic films of the same thickness $d_{\mathrm{m}}$, which are separated by a nonmagnetic film of thickness $d$. The electronic properties are described within the free-electron-like approximation with a spin dependent effective electron potential $U_{\sigma}(z)$. The potential is uniform across the layers and inside the magnetic films $U_{\sigma}(z)=U_{+}$if $\sigma$ corresponds to the spin-majority electrons and $U_{\sigma}(z)=U_{-}$for the spin-minority electrons. In the nonmagnetic spacer we assume $U_{\sigma}(z)=U_{0}=0$ for both spin orientations.

When considering only parallel and antiparallel orientations of the film magnetizations (and neglecting spin-flip scattering processes), one may analyse both spin channels separately and simply add the conductivities of the individual channels. Taking into account the Kubo formula one can write the leading contribution to the global in-plane conductivity $g$ in the form

$$
g=\frac{1}{2 L}\left(\frac{e}{m}\right)^{2} \frac{\hbar}{(2 \pi)^{2}} \sum_{\sigma} \int_{0}^{q \mathrm{~F}} \mathrm{~d} q \int_{0}^{L} \mathrm{~d} z \int_{0}^{L} \mathrm{~d} z^{\prime} q^{3} G^{\mathrm{R}}{ }_{q \sigma}\left(z, z^{\prime}\right) G_{q \sigma}^{\mathrm{A}}\left(z^{\prime}, z\right),
$$

where $q_{\mathrm{F}}$ is the Fermi wave vector and $L$ is the total thickness of the structure. The retarded and advanced Green functions, $G^{\mathrm{R}}{ }_{q \sigma}\left(z, z^{\prime}\right)$ and $G^{\mathrm{A}}{ }_{q \sigma}\left(z, z^{\prime}\right)$, are written here in the mixed $(q, z)$ representation and are taken at the Fermi energy $E_{\mathrm{F}}$.

The impurity averaged Green function $G^{\mathrm{R}}{ }_{q \sigma}\left(z, z^{\prime}\right)$ can be found from the differential equation

$$
\left(\frac{\hbar^{2}}{2 m} \frac{\mathrm{d}^{2}}{\mathrm{~d} z^{2}}+E_{\mathrm{F}}-\frac{\hbar^{2} q^{2}}{2 m}-U_{\sigma}(z)+\mathrm{i} \frac{\hbar}{2 \tau_{\sigma}(z)}\right) G_{q \sigma}^{\mathrm{R}}\left(z, z^{\prime}\right)=\hbar \delta\left(z-z^{\prime}\right),
$$

where $\tau_{\sigma}(z)$ is the local relaxation time. The calculations are significantly simplified by assuming $\tau_{\sigma}(z)$ constant across each sublayer, but different in different layers. This is, however, a very rough approximation. It does not lead to quantum-size oscillations of the conductivity in a single film when the number of particles is constant. It also gives no oscillations due to external size effects in sandwich structures. The only oscillatory term which survives (at least in a changed form) is the one due to internal size effects. Thus the approximation may be used in the limit where long-period oscillations in GMR dominate. The advantage of this approximation is that it enables analytical derivation of the corresponding Green functions and, consequently, analytical integrations over $z$ and $z^{\prime}$ in Eq. (1). The integration over $q$; however, has to be performed numerically.

To calculate the interlayer coupling we neglect the influence of the impurities. The electronic spectrum consists then of two-dimensional parabolic minibands and the coupling parameter $J$ is given by [8]

$$
J=\frac{m}{4 \pi \hbar^{2}}\left[\left(E_{\mathrm{F}}^{2} N-\Sigma\right)_{\uparrow \downarrow}-\left(E_{\mathrm{F}}^{2} N-\Sigma\right)_{\uparrow \uparrow}\right] .
$$

Here, $N$ is the number of occupied minibands, $N=\sum_{n \sigma} \Theta\left(E_{\mathrm{F}}-\epsilon_{n \sigma}\right)$ and $\Sigma$ is defined as $\Sigma=\sum_{n \sigma} \epsilon_{n \sigma}^{2} \Theta\left(E_{\mathrm{F}}-\epsilon_{n \sigma}\right)$, where $\epsilon_{n \sigma}$ are the discrete energy levels for 

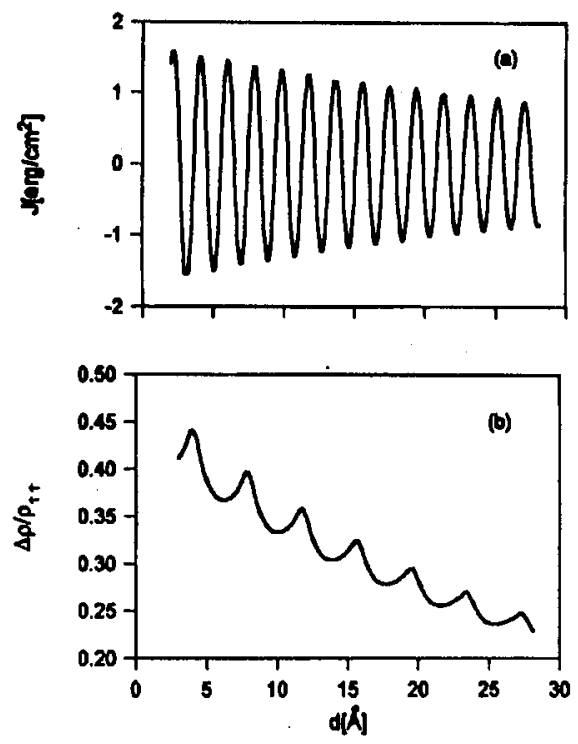

Fig. 1. The coupling parameter $J$ (a) and the ratio $\Delta \rho / \rho_{\uparrow \uparrow}$ (b) as a function of the spacer thickness $d$ calculated for $d_{\mathrm{m}}=30 \AA, \lambda_{+}=295 \AA, \lambda_{-}=1709 \AA, \lambda=$ $522 \AA, U_{-}=2.43 \mathrm{eV}, U_{+}=1.92 \mathrm{eV}$ and the corresponding Fermi energy in the bulk limit equal to $10.27 \mathrm{eV}$.

spin $\sigma$, whereas $\Theta(x)=1$ for $x \geq 0$ and $\Theta(x)=0$ for $x<0$. The two terms on the right side of Eq. (3) are to be calculated in the antiparallel and parallel configuration of the film magnetizations.

Numerical results for a constant particle number are shown in Fig. 1, where the coupling parameter $J$ and the relative resistivity change, $\Delta \rho / \rho_{\uparrow \uparrow}=$ $\left(\rho_{\uparrow \downarrow}-\rho_{\uparrow \uparrow}\right) / \rho_{\uparrow \uparrow}\left(\rho_{\uparrow \downarrow}\right.$ and $\rho_{\uparrow \uparrow}$ being the resistivities respectively in the antiparallel and parallel configurations) are shown as a function of the spacer thickness. The GMR effect is there due to spin dependent electron scattering on impurities inside the magnetic films, which is described by the electron mean free paths $\lambda_{+}$and $\lambda_{-}$ respectively for the spin-majority and spin-minority electrons. For the nonmagnetic spacer we assume the same mean free path $\lambda$ for both spin orientations. The oscillations in GMR originate from the internal size effect and the corresponding oscillation period depends on the potential barriers at the interfaces. The short-period $\left(\Lambda_{c}\right)$ oscillations do not occur in GMR in the approximation used. However, only the short-period oscillations are allowed in the coupling parameter. Thus, when the long-period oscillations in GMR dominate, then the oscillations in GMR and interlayer coupling are different and not correlated. For the parameters used in Fig. 1 the oscillation periods differ by a factor approximately equal to 2, but in general this factor can be arbitrary. 


\section{Acknowledgments}

The work was supported by the Committee for Scientific Research through the research project 2 P03B 165 10. J.B. also acknowledges support from the Belgian Interuniversity Attraction Poles and Flemish Concerted Action Programs.

\section{References}

[1] S.S.P. Parkin, N. More, K.P. Roche, Phys. Rev. Lett. 64, 2304 (1990).

[2] Y. Yafet, Phys. Rev. B 36, 3948 (1987); P. Bruno, C. Chappert, Phys. Rev. Lett. 67, 1602 (1991).

[3] M.D. Stiles; Phys. Rev. B 48, 7238 (1993).

[4] M.N. Baibich, J.M. Broto, A. Fert, F. Nguyen van Dau, F. Petroff, P. Etienne, G. Creuzet, A. Friederich, J. Chazelas, Phys. Rev. Lett. 61, 2472 (1988).

[5] A. Vedyayev, C. Cowache, N. Ryzhanova, B. Dieny, J. Phys., Condens. Matter 5, 8289 (1993).

[6] B. Bułka, J. Barnaś, Phys. Rev. B 51, 6348 (1995).

[7] J. Barnaś, Y. Bruynseraede, Phys. Rev. B 53, R2956 (1996).

[8] J. Barnaś, Phys. Rev. B 52, 10744 (1995). 